\title{
Évaluation des contraintes résiduelles induites par soudage par la méthode ultrasonore : analyse de l'effet de la microstructure
}

\author{
Jazzar Hoblos $^{1,2, a}$, Gérard Bourse ${ }^{1}$, Claude Robin $^{1}$, Henri Walaszek $^{2}$ \\ et Henri-Paul Lieurade ${ }^{2}$ \\ 1 Département Mécanique et Comportement des Matériaux, École des Mines de Douai, 941 rue Charles Bourseul, \\ 59508 Douai, France \\ 2 Centre Technique des Industries Mécaniques, 52 Av. Félix Louat, 60304 Senlis, France
}

Reçu le 8 juin 2005, accepté le 3 février 2006

\begin{abstract}
Résumé - L'utilisation de la méthode ultrasonore pour l'évaluation des contraintes résiduelles repose sur l'effet acoustoélastique qui traduit une dépendance de la vitesse de propagation des ondes acoustiques en fonction de l'état de déformation du solide. Dans le cas du soudage, les modifications microstructurales observées dans la zone affectée thermiquement et dans la zone fondue induisent également des variations de la vitesse de propagation des ondes acoustiques. La superposition des deux effets : contraintes et microstructure conduit à surestimer les niveaux de contraintes. Ce travail réalisé en collaboration avec le CETIM de Senlis essaie d'apporter une réponse à ce problème observé dans le cas du soudage. La solution adoptée consiste à caractériser l'effet acoustoélastique ainsi que les vitesses de propagation $V_{0}$ pour les différentes zones de la soudure. L'étude expérimentale effectuée sur des tôles en aciers P460 HLE et P265 soudées avec un chanfrein en $\mathrm{X}$ a permis de valider notre démarche. Ceci nous permet d'envisager sous certaines conditions, l'utilisation de cette méthode dans le cas des équipements sous pression.
\end{abstract}

Mots clés : Contraintes résiduelles / méthode ultrasonore / onde $L_{\mathrm{cr}}$ / effet de la microstructure / plaque soudée / effet acoustoélastique

\begin{abstract}
Evaluation of residual stresses induced by welding by using ultrasonic method: analysis of microstructure effect. The use of the ultrasonic method for the evaluation of the residual stresses is based on the acoustoelastic effect which refers to the change in velocity of the acoustic waves propagating in a strained solid. In the case of welding, the microstructural modifications observed in the heat affected zone and the molten zone also induce variations of the velocity of the acoustic waves. The superposition of the two effects: stresses and microstructure results in over-estimating the levels of stresses. This work completed in collaboration with CETIM of Senlis is a contribution to this problem. The solution proposed consists in characterizing the acoustoelastic effect as well as the propagation velocities $V_{0}$ for the different welded zones. The experimental study carried out on welded sheets with a chamfer in $\mathrm{X}$ on P460 HLE and P265 steel, which enables to consider, that under certain conditions, the use of this method in the case of pressure equipments is possible.
\end{abstract}

Key words: Residual stresses / ultrasonic method / $L_{\mathrm{cr}}$ waves / microstructure effects / welded plates / acoustoelastic effect

\section{Introduction}

Les différentes opérations de transformation des matériaux utilisées dans l'industrie mécanique induisent des contraintes résiduelles. Celles-ci peuvent être volontairement introduites pour améliorer les caractéristiques

\footnotetext{
${ }^{a}$ Auteur correspondant : jazzar_hoblos@yahoo.fr
}

mécaniques (grenaillage, traitements thermiques superficiels) ou involontairement (soudage, meulage, rectification). Dans ce cas elles sont souvent défavorables à la tenue mécanique de la pièce et doivent être prises en compte lors de la conception et du dimensionnement afin de ne pas diminuer la durée de vie de celle-ci. La connaissance de l'état de contraintes résiduelles revêt donc une réelle importance pour les pièces mécaniques. 
Il existe différentes techniques permettant de déterminer ces contraintes résiduelles : la méthode de diffraction des rayons $\mathrm{X}$, la méthode du trou (méthode semi-destructive), la méthode Barkhausen basée sur les propriétés ferromagnétiques du matériau et la méthode ultrasonore. La méthode de diffraction des rayons $\mathrm{X}$ permet de mesurer la déformation du réseau interréticulaire occasionnée par la présence des contraintes. La mesure de la distance entre plans $(h k l)$ de mêmes indices permet de remonter aux contraintes macroscopiques présentes dans la pièce. $\mathrm{Vu}$ la faible pénétration des rayons $\mathrm{X}(\approx 10 \mu \mathrm{m}$ pour l'acier), on obtient par cette méthode des mesures de surface. Des profils de contraintes sont possibles dans l'épaisseur, mais dans ce cas la méthode devient semidestructive. La méthode du trou repose sur la relaxation des contraintes qui est obtenue lors du perçage d'un trou dans la pièce. Cette relaxation est mesurée à l'aide de jauges d'extensométrie. Il est également possible de déterminer le gradient de contraintes dans l'épaisseur. Ces deux méthodes sont bien établies alors que les méthodes Barkhausen et ultrasonore sont toujours en cours d'étude.

Depuis plusieurs années, l'École des Mines de Douai et le CETIM de Senlis travaillent en collaboration sur la thématique concernant l'évaluation non-destructive des contraintes appliquées ou résiduelles par méthode ultrasonore. Le travail présenté dans cet article concerne l'évaluation des contraintes de soudage et essaie de répondre au problème du changement de microstructure au voisinage du cordon soudé.

\section{Détermination des contraintes résiduelles par méthode ultrasonore}

\subsection{Principe de la méthode}

La détermination des contraintes par méthode ultrasonore repose sur l'effet acoustoélastique, qui se traduit par des variations de vitesses de propagation des ondes acoustiques en fonction de l'état de déformation du matériau. Dès les années 1950, on voit apparaître les premiers résultats concernant la caractérisation de contraintes appliquées dans les matériaux polycristallins. Le formalisme qui permet d'expliquer l'effet acoustoélastique est basé sur la théorie développée par Murnaghan [1] sur les déformations finies dans les solides élastiques qui repose sur des phénomènes d'élasticité non-linéaire. Cette théorie fait intervenir des termes d'ordre trois dans la fonction d'énergie de déformation qui conduisent à des non-linéarités dans la loi de comportement du matériau. En plus des deux constantes élastiques $(\lambda, \mu)$ classiques, trois autres constantes $(l, m, n$ : constantes du troisième ordre de Murnaghan) sont nécessaires pour décrire le comportement d'un matériau isotrope. De nombreux travaux théoriques [2-4] ont été publiés sur le sujet, les plus récents notamment ceux de Bray [5-9] concernent des applications industrielles. On peut également noter que cette méthode est utilisée pour l'évaluation des contraintes dans les rails et les roues de matériel ferroviaire ainsi que pour déterminer le serrage des assemblages boulonnés.

Hughes et Kelly [2] ont utilisé le modèle de Murnaghan afin de résoudre l'équation d'onde dans le cas du solide déformé. Ils ont exprimé les solutions donnant les vitesses de propagation des ondes planes qui se propagent dans un milieu isotrope :

$$
\begin{aligned}
& \rho_{0} V_{11}^{2}=\lambda+2 \mu+(2 \ell+\lambda) \theta+(4 m+4 \lambda+10 \mu) \alpha_{1} \\
& \rho_{0} V_{12}^{2}=\mu+(\lambda+m) \theta+4 \mu \alpha_{1}+2 \mu \alpha_{2}-\frac{1}{2} n \alpha_{3} \\
& \rho_{0} V_{13}^{2}=\mu+(\lambda+m) \theta+4 \mu \alpha_{1}+2 \mu \alpha_{3}-\frac{1}{2} n \alpha_{2}
\end{aligned}
$$

avec $: \rho_{0}:$ masse volumique initiale du matériau de propagation

$V_{i j}$ : vitesse de l'onde se propageant suivant l'axe $i$ et polarisée selon l'axe $j$.

$\lambda$ et $\mu$ : coefficients du second ordre de Lamé.

$l, m, n$ : constantes du troisième ordre de Murnaghan.

$\theta=\alpha_{1}+\alpha_{2}+\alpha_{3}$ : trace de la matrice des déformations.

\subsection{Application au cas d'un chargement uniaxial}

Dans le cas d'une contrainte de traction $(\sigma=E . \varepsilon)$ suivant 1 , on peut écrire : $\alpha_{1}=\varepsilon, \alpha_{2}=\alpha_{3}=-\nu$. $\varepsilon$

En injectant dans l'équation (1) les valeurs des déformations principales, on obtient :

$\rho_{0} V_{11}^{2}=\lambda+2 \mu+\left[4(\lambda+2 \mu)+2(\mu+2 m)+\nu \mu\left(1+\frac{2 l}{\lambda}\right)\right] . \varepsilon$

On peut ensuite exprimer la variation relative de vitesse en fonction de la déformation pour chacun des modes, l'expression suivante correspond au mode longitudinal :

$$
\frac{\frac{\mathrm{d} V_{11}}{V_{11}}}{\mathrm{~d} \varepsilon}=2+\frac{(\mu+2 m)+\nu \mu\left(1+\frac{2 l}{\lambda}\right)}{\lambda+2 \mu}=A_{11}
$$

où $A_{11}$ est la constante acoustoélastique correspondant au mode longitudinal, elle est fonction de : $\lambda, \mu, l, m$. Les constantes acoustoélastiques des modes transversaux sont fonction de $: \lambda, \mu, m, n$.

Les constantes acoustoélastiques doivent nécessairement être déterminées pour le matériau utilisé et elles sont sensibles à sa microstructure. Elles sont obtenues en mesurant la variation relative de vitesse du mode utilisé lors d'un essai de traction (Fig. 1).

\section{Méthode expérimentale}

\subsection{Les échantillons utilisés}

Nous avons travaillé sur deux aciers de construction : P460 HLE et P265. Les tôles ayant une épaisseur de 


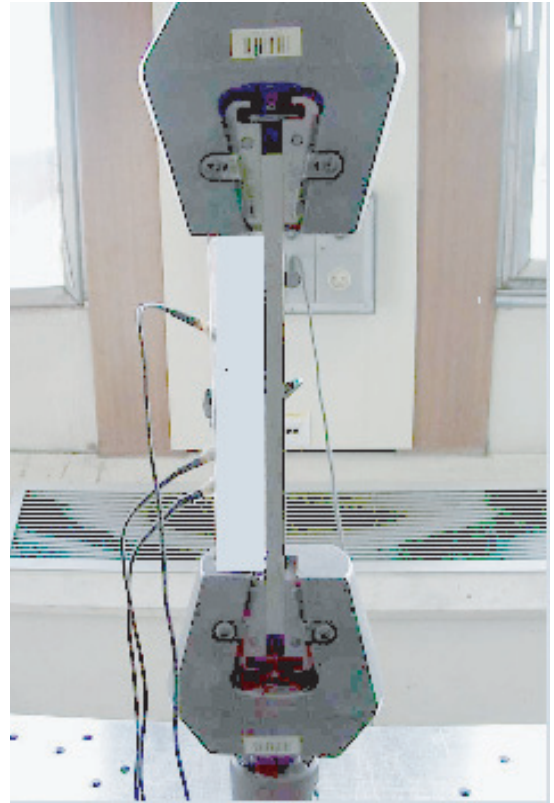

Fig. 1. essai de traction dans la zone fondue.

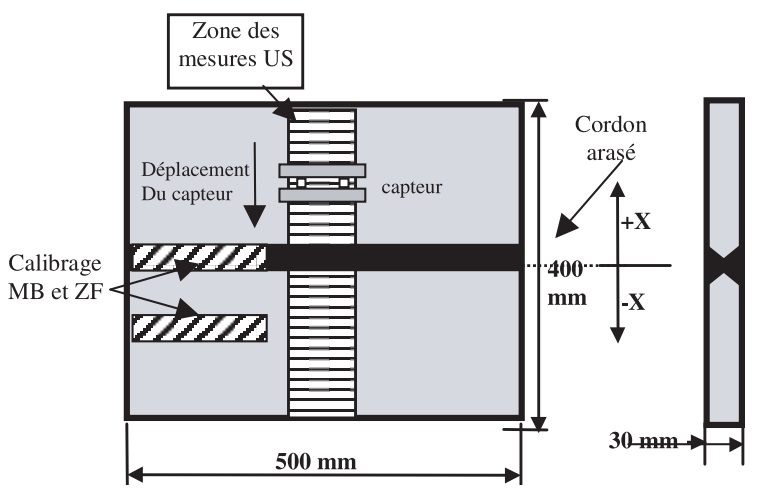

Fig. 2. Tôles soudées utilisées pour l'étude.

$30 \mathrm{~mm}$ sont soudées avec un chanfrein en $\mathrm{X}$ avec le même métal d'apport. Le soudage est manuel pour la tôle en acier P460, il est automatique sous flux pour la tôle en acier P265. La surface de mesure a été rectifiée avec des passes fines de 5 micro pour ne pas modifier les contraintes. La figure 2 présente le schéma des tôles utilisées.

\subsection{Le système de mesure}

Lorsqu'il existe un gradient de contrainte dans l'épaisseur, l'utilisation des ondes de volume longitudinales ou transversales ne permet d'obtenir qu'une évaluation de la contrainte moyenne dans l'épaisseur. Elles sont généralement utilisées lorsque la contrainte est homogène dans l'épaisseur, comme par exemple lors de l'évaluation du serrage des assemblages boulonnés ou pour évaluer les contraintes dans le bandage des roues de matériel ferroviaire.

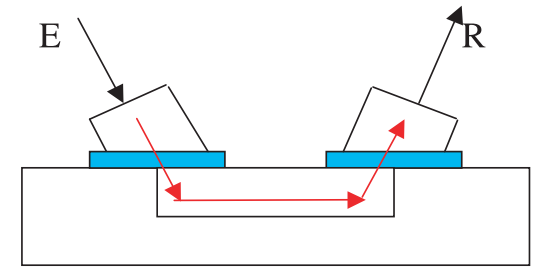

Fig. 3. Schéma de principe du capteur.

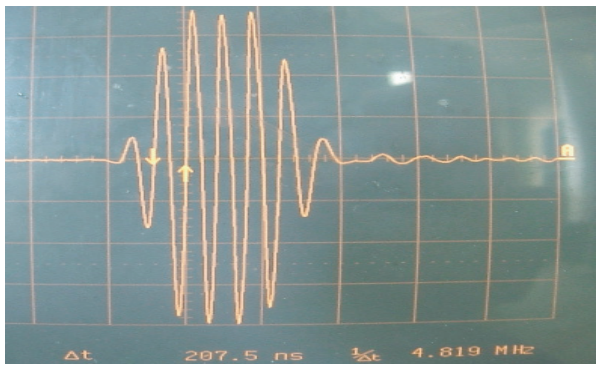

(a)

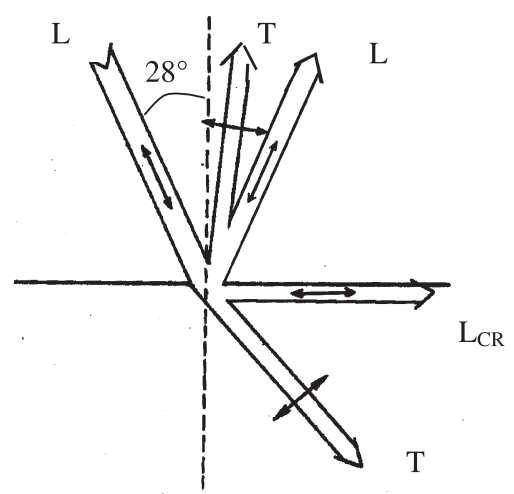

(b)

Fig. 4. a) Signal du mode $L_{\mathrm{CR}}$. b) $L_{\mathrm{cr}}$ (critically refracted longitudinal wave).

C'est pourquoi dans le cas des contraintes induites par soudage, nous avons privilégié l'emploi des ondes rampantes $\left(L_{\mathrm{CR}}\right)$ figure 4 , qui sont des ondes longitudinales se propageant sur une profondeur qui est de l'ordre d'une longueur d'onde, soit environ 1,3 à $3 \mathrm{~mm}$.

Ces ondes sont produites par réfraction au moyen de prismes en « PMMA ». Les capteurs (Fig. 3) sont composés de deux éléments (1 émetteur et 1 récepteur formant un bloc rigide), ils ont l'avantage de fixer le trajet des ondes et donc de ramener la mesure de vitesse à une mesure de temps. Les mesures de temps (Fig. 4a) sont effectuées en utilisant la méthode du passage à zéro sur une rampe positive du signal. Cette méthode permet d'obtenir des mesures de temps très précises $( \pm 1 \mathrm{~ns})$. Le système de mesure est présenté à la figure 5 , il comprend un générateur d'impulsions qui permet d'exciter l'émetteur et d'amplifier les signaux reçus par le récepteur. Les signaux sont ensuite échantillonnés par une carte d'acquisition numérique (interface IEEE). Le traitement des 


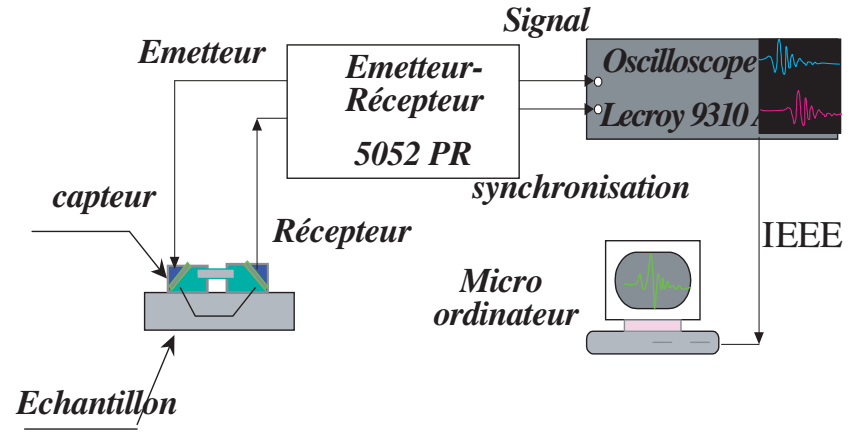

Fig. 5. Système de mesure des temps de propagation.

signaux est effectué par un micro-ordinateur à l'aide d'un logiciel développé sous Labview.

\subsection{Le calibrage de l'effet acoustoélastique}

Pour utiliser la méthode ultrasonore, il est nécessaire de connaître les constantes acoustoélastiques du matériau. Ces constantes sont obtenues par l'intermédiaire d'un calibrage effectué à partir d'un essai de traction, afin de déterminer la constante de proportionnalité reliant la variation relative de vitesse de l'onde et la contrainte appliquée, selon une loi pouvant s'exprimer de la manière suivante :

$$
\frac{\mathrm{d} V}{V_{0}}=K * \sigma \text { avec } K=\frac{A_{11}}{E} \text { pour le mode } L_{\mathrm{CR}}
$$

avec $E$ : Module d'élasticité du matériau $A_{11}$ d'après (3)

Cette expression est équivalente à :

$$
V(\sigma)=V_{0}(1+K * \sigma)
$$

L'opération de soudage entraîne, à cause de l'apport de chaleur dans le matériau et de la vitesse de refroidissement, une évolution de la microstructure entre le métal de base (MB) et la zone fondue (ZF), on peut ainsi généralement constater l'évolution d'une structure ferrito-perlitique (MB) vers une structure bainitique ou bainite-martensite (ZF). Dans le cas du soudage, il s'avère nécessaire de déterminer la constante $K$ (Fig. 6a) dans le métal de base et dans la zone fondue, il n'est pas possible de le faire dans la zone affectée thermiquement (ZAT) en raison de sa faible dimension (3 à $4 \mathrm{~mm}$ ). Le double calibrage est nécessaire car les constantes acoustoélastiques dépendent de la microstructure du matériau.

\section{Évaluation des constantes acoustoélastiques}

La figure 7 présente le résultat du calibrage concernant un acier P460 qui a été effectué dans les deux zones, c'està-dire le métal de base et la zone fondue.

Il y a bien un effet dû à la microstructure puisque nous obtenons deux valeurs distinctes de constantes acoustoélastiques. On vérifie (cf. Tab. 1) le même comportement pour l'acier P265, et l'on remarque une différence

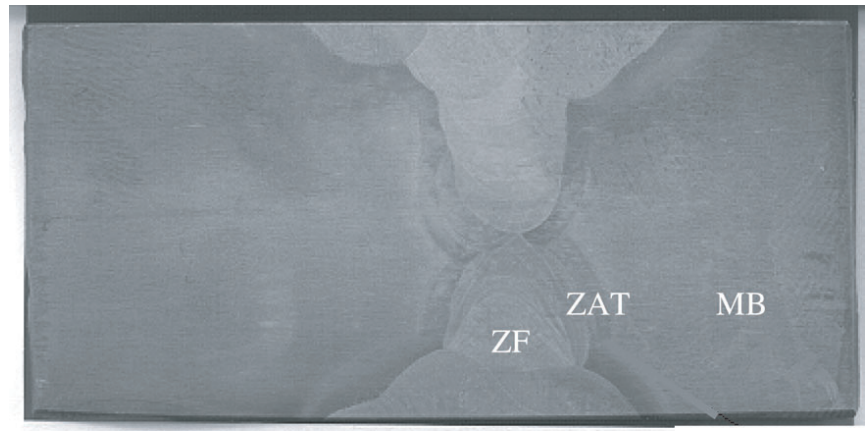

a)

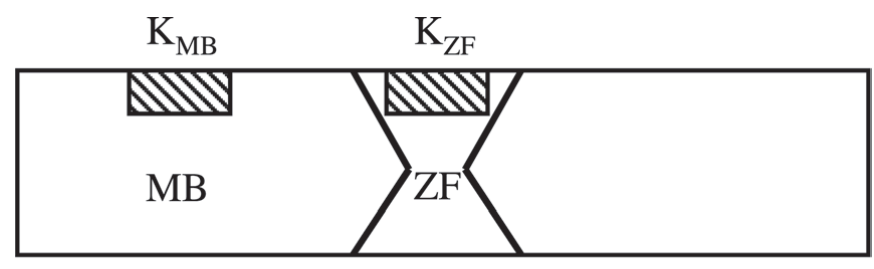

MIIV Echantillons prélevés pour le calibrage

b)

Fig. 6. Structure de la soudure de l'acier P460. b) Réalisation du double calibrage acoustoélastique.

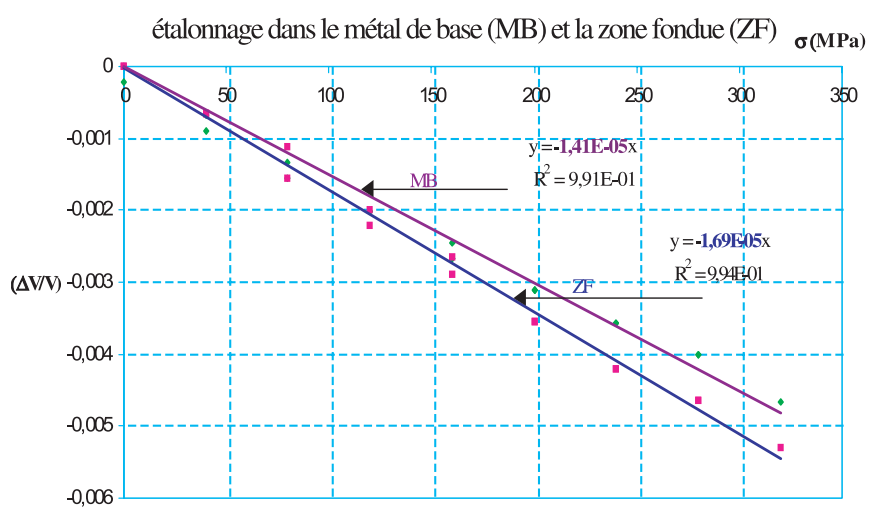

Fig. 7. Calibrage de l'effet acoustoélastique sur l'acier P460.

Tableau 1. Valeurs des constantes acoustoélastiques dans le métal de base et la zone fondue.

\begin{tabular}{ccc}
\hline & $K_{11 \mathrm{PM}}$ & $K_{11 \mathrm{MZ}}$ \\
\hline P460 HLE & $-1.41 \times 10^{-05}$ & $-1.69 \times 10^{-05}$ \\
P265 & $-1.33 \times 10^{-05}$ & $-1.48 \times 10^{-05}$ \\
\hline
\end{tabular}

des valeurs de $K_{\text {ZF }}$ pour les soudures effectuées en mode manuel et automatique.

L'analyse des résultats de calibrage de l'effet acoustoélastique (cf. Tab. 1) montre pour les cas étudiés que la valeur de la constante acoustoélastique dépend à la fois du matériau et de la zone de soudage, mais que le rapport des constantes $K_{\mathrm{ZF}} / K_{\mathrm{MB}}$ ne dépend que du procédé de soudage utilisé [10]. 


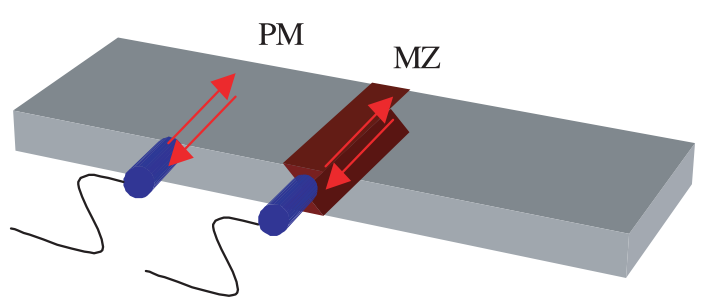

(a)

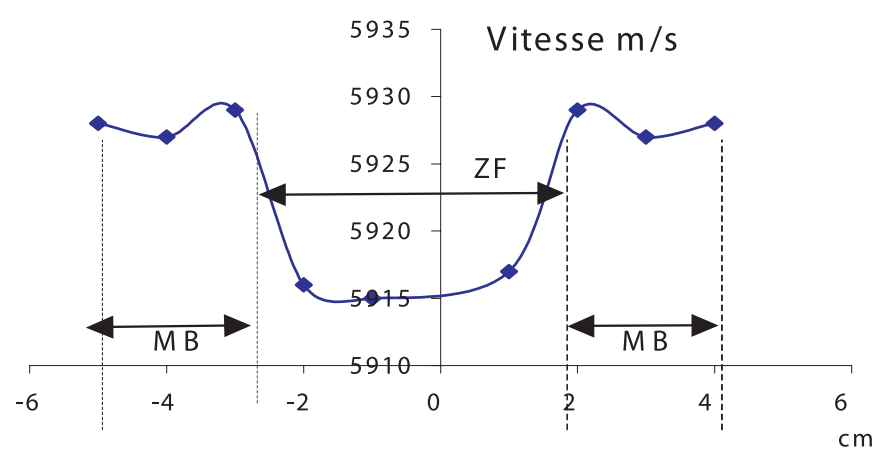

(b)

Fig. 8. a) Méthode de mesure de la vitesse longitudinale. b) Mesure de la vitesse longitudinale à contrainte nulle (tôle soudée P460).

\subsection{Principe de la mesure}

Les mesures de variations de vitesse sont ramenées à des variations du temps de propagation $(\mathrm{d} t)$ grâce à la conception du capteur qui impose à l'onde un trajet fixe. La mesure $\mathrm{d} u \mathrm{~d} t\left(t-t_{0}=\mathrm{d} t\right)$ est obtenue en comparant le temps de propagation $t_{0}$ de l'onde dans une zone réputée sans contraintes avec celui $(t)$ qui est mesuré dans une zone où s'exercent les contraintes. À partir des mesures de $\mathrm{d} t$, il est alors possible de remonter à la valeur de la contrainte en utilisant l'expression suivante :

$$
\mathrm{d} \sigma=\frac{E}{A_{11}}\left(\frac{\mathrm{d} V_{11}}{V_{11}^{0}}\right)=-\frac{1}{K} * \frac{\mathrm{d} t}{t_{0}}
$$

où $K$ est la constante acoustoélastique déterminée par calibrage.

Dans le cas des aciers ferritiques, il n'y a pas d'effet de la contrainte perpendiculaire à la direction de propagation. On peut ainsi déterminer la valeur de la contrainte dans la direction de propagation à partir de l'expression (6). Selon la zone de mesure, il sera nécessaire d'utiliser $K_{\mathrm{MB}}$ pour le métal de base, $K_{\mathrm{ZF}}$ pour la zone fondue. Il faut également prendre en compte l'effet de la microstructure sur les vitesses de propagation $V_{0 \mathrm{MB}}$ et $V_{0 \mathrm{ZF}}(\sigma=0)$ afin de déterminer les temps de vol de référence $\left(t_{0}\right)$ dans les deux zones. Nous avons préparé deux échantillons prélevés sur les tôles et nous les avons détentionnés en dessous de $600{ }^{\circ} \mathrm{C}$ pour éliminer les contraintes et garder la microstructure initiale. Les valeurs de $\left(K, t_{0}\right)$ propres à chaque zone de mesure devront être injectées dans l'expression (6) afin de calculer les valeurs de contrainte. La figure 8 montre l'évolution de la
Tableau 2. Détermination des vitesses à contrainte nulle dans les deux zones MB et ZF.

\begin{tabular}{cccc}
\hline Acier & $V_{\text {OZF }} / V_{0 \mathrm{MB}}$ & $V_{0 \mathrm{MB}}\left({\mathrm{m} . \mathrm{s}^{-1}}\right)$ & $V_{0 \mathrm{ZF}}\left(\mathrm{m} . \mathrm{s}^{-1}\right)$ \\
\hline P460 & 0,998 & 5928 & 5916 \\
P265 & 0,998 & 5932 & 5923 \\
(tôle) & & & \\
\hline
\end{tabular}

vitesse de l'onde longitudinale mesurée sur l'échantillon en acier P460 dans la direction parallèle à l'axe du cordon lorsqu'on passe du métal de base à la zone affectée.

Le tableau 2 regroupe les différentes valeurs de vitesses $V_{0}(\sigma=0)$ dans le métal de base et la zone fondue pour les deux aciers étudiés.

Les niveaux des contraintes sont obtenus en prenant en compte les paramètres liés à chaque zone (MB, ZF), comme c'est indiqué dans les équations suivantes :

$$
\begin{aligned}
\sigma_{\mathrm{ZF}}=-\frac{1}{K_{\mathrm{ZF}}} * \frac{\left(t-t_{0 \mathrm{ZF}}\right)}{t_{0 \mathrm{ZF}}} \text { et } & \\
& \sigma_{\mathrm{MB}}=-\frac{1}{K_{\mathrm{MB}}} * \frac{\left(t-t_{0 \mathrm{MB}}\right)}{t_{0 \mathrm{MB}}}
\end{aligned}
$$

L'analyse des résultats concernant la détermination des vitesses $V_{0}$ dans les différentes zones de la soudure (cf. Tab. 2) montre que la valeur du rapport $V_{0 \mathrm{ZF}} / V_{0 \mathrm{MB}}$ est indépendante du matériau, de la zone et du procédé de soudage. Ceci est très intéressant, car nous avons constaté précédemment (Tab. 1) que le rapport $K_{\mathrm{ZF}} / K_{\mathrm{MB}}$ ne dépendait que du procédé de soudage. Ces deux résultats nous permettent de penser que l'on pourra limiter par la suite les calibrages dans le cas d'une application industrielle de la méthode.

\section{Résultats expérimentaux}

Les mesures ont été réalisées avec des capteurs émettant des ondes longitudinales de sub-surface appelées $L_{\mathrm{CR}}$. Les fréquences utilisées sont 2 et $5 \mathrm{MHz}$ pour les tôles en acier P460 et P265, les profondeurs de pénétration sont de 3 et 1,3 $\mathrm{mm}$ respectivement. Les dimensions des capteurs sont (carré, $8 \mathrm{~mm}, 2 \mathrm{MHz}$ ) et (circulaire, $5 \mathrm{~mm}, 5 \mathrm{MHz}$ ). Les profils de contraintes présentés dans cette étude concernent uniquement la contrainte longitudinale $\sigma_{\mathrm{L}}$, qui s'exerce parallèlement à l'axe du cordon.

\subsection{Acier P460 HLE}

Le premier résultat concerne une tôle d'épaisseur $30 \mathrm{~mm}$ soudée bout à bout avec un chanfrein en $\mathrm{X}$ réalisée en acier P460 HLE. Les mesures ont été réalisées 4 fois afin de s'assurer de la reproductibilité des résultats. La figure 9 présente les profils de contraintes obtenus au voisinage du cordon soudé avant et après prise en compte des constantes acoustoélastiques $K_{\mathrm{MB}}$ et $K_{\mathrm{ZF}}$ propres à chacune des deux zones de la soudure ainsi que des vitesses 


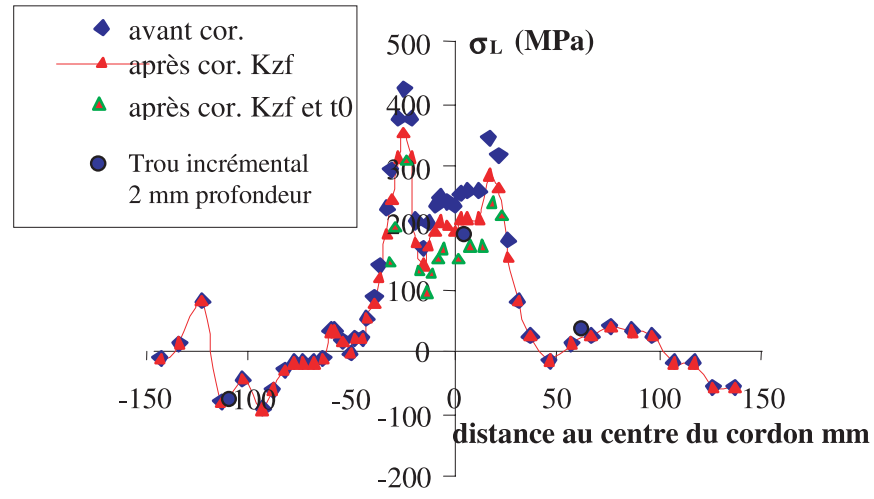

Fig. 9. Profil des contraintes sur plaque en acier $\mathrm{P} 460$ à $2 \mathrm{MHz}$ (soudure mode manuel).

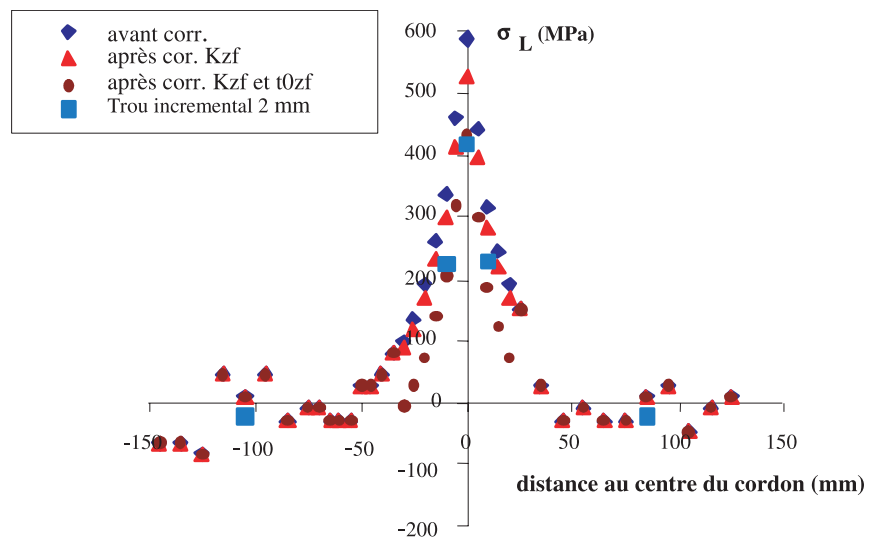

Fig. 10. Profil des contraintes sur plaque en acier P265 à $2 \mathrm{MHz}$ (soudure mode automatique).

$V_{0 \mathrm{MB}}$ et $V_{0 \mathrm{ZF}}$. Cette méthode entraîne une diminution de la valeur des contraintes comprise entre 15 et $100 \mathrm{MPa}$ dans la zone fondue.

\subsection{Acier P265}

L'exemple suivant présenté à la figure 10 concerne une tôle soudée en acier P265 du même type que la précédente. Les mesures ultrasonores ont été effectuées à la fréquence de $2 \mathrm{MHz}$. La correction concernant la constante acoustoélastique $K_{\mathrm{ZF}}$ et celle sur la vitesse de propagation $V_{0 Z F}$ ont été effectuées de manière à prendre en compte l'effet dû à la différence de microstructure entre le métal de base et la zone fondue. Cette correction agit sur le temps de propagation de l'onde $t_{0 \mathrm{ZF}}$ servant de référence pour un parcours effectué dans une zone libre de contraintes. La double correction entraîne une diminution de la valeur des contraintes comprise entre 100 et $150 \mathrm{MPa}$ dans la zone fondue. Ces valeurs de contraintes sont supérieures à la limite d'élasticité (309 MPa) du métal de base mais sont équivalentes à celles déterminées par la méthode du trou.

\section{Conclusion}

Ce travail montre la possibilité d'évaluer les contraintes induites par soudage en utilisant la méthode ultrasonore. L'utilisation des ondes $L_{\mathrm{CR}}$ permet de remonter directement à la contrainte qui s'exerce suivant la direction de propagation. Ainsi, les mesures effectuées en positionnant le capteur parallèlement à l'axe du cordon de soudure nous ont permis de déterminer la contrainte longitudinale $\sigma_{\mathrm{L}}$. Ceci est possible dans le cas des aciers, car l'onde $L_{\mathrm{CR}}$ est peu sensible à la présence des contraintes qui s'exercent suivant une direction perpendiculaire à celle de sa propagation.

Le problème rencontré lors de l'utilisation de cette méthode concerne la séparation de l'effet relatif à la microstructure de celui dû à la présence des contraintes dans le joint soudé. Pour répondre à ce problème, nous avons proposé de déterminer les constantes acoustoélastiques ainsi que les vitesses $V_{0}(\sigma=0)$ pour chacune des zones (MB et $\mathrm{ZF}$ ) de la soudure. Ces paramètres sont obtenus à partir de calibrages qui ont été décrits dans ce travail. L'analyse des résultats obtenus sur deux aciers soudés avec deux procédés de soudage a permis de dégager les points suivants :

- Le rapport des constantes acoustoélastiques $K_{\mathrm{ZF}} / K_{\mathrm{MB}}$ ne dépend que du procédé de soudage

- Le rapport des vitesses $V_{0 \mathrm{ZF}} / V_{0 \mathrm{MB}}$ est indépendant du matériau et du procédé de soudage

Ces résultats sont intéressants dans l'optique d'une application industrielle de la méthode, car on peut envisager de limiter les calibrages lorsqu'on change de type d'acier.

- Les contraintes qui ont été déterminées pour les aciers étudiés sont de traction, ceci peut être relié à la contraction du joint soudé lors du refroidissement.

- Les niveaux de contraintes déterminés dans le cordon sont plus élevés (100 à $200 \mathrm{MPa}$ ) dans le cas des soudures automatiques.

\section{Références}

[1] T.D. Murnaghan, Finite Deformation of an elastic solid, John Wiley, New York, 1951

[2] D.S. Hughes, J.L. Kelly, Second-order elastic deformation of solids, Phys. Rev. 92 (1953) 1145-1149

[3] D.M. Egle, Bray, D. E., Measurement of acoustoelastic and third-order elastic constants for rail steels, J.A.S.A 60 (1976) 741-744

[4] E. Tanala, G. Bourse, M. Fremiot, J.F. De Belleval, Determination of near surface residual stresses on welded joints using ultrasonic methods, NDT \& E International 28 (1995) 83-88

[5] D.E. Bray, Ultrasonic stress measurement and material characterization in pressure vessels, piping, and welds, J. Pressure Vessel Technology 124 (2002) 326-335

[6] H. Walaszek, H.P. Lieurade, C. Peyrac, J. Hoblos, J. Rivenez, Potentialities of ultrasonics for evaluating residual stresses: Influence of microstructure, J. Pressure Vessel Technology 124 (2002) 349-353 
[7] D.E. Bray, Current directions of Ultrasonic Stress Measurement Techniques, 15th WCNDT, Rome, 2000, http://www.ndt.net/article/wcndt00/papers/idn647/ idn647.htm

[8] D.E. Bray, W. Tang, D.S. Grewal, Ultrasonic Stress Evaluation in a Compressor Rotor, J. Testing and Evaluation 25 (1997) 503-509

[9] J. Hoblos, G. Bourse, C. Robin, H. Walaszek, S. Chaki, Ultrasonic stress measurement in welded joints by using $L_{\mathrm{CR}}$ Waves: An approach to separate microstructure and stress effects, WCU 2003, Paris, September 7-10, 2003, pp. 701-704

[10] H. Walaszek, J. Hoblos, G. Bourse, C. Robin, P. Bouteille, H.P. Lieurade, Ultrasonic stress measurement in welded component by using $L_{\mathrm{cr}}$ waves: analysis of the microstructure effect, ASME, PVP volume 484, recent advances in Non destructive evaluation technique for material service and industry 25-29 juillet 2004 San Diego Cal. Usa, article 28-29 pp. 147-152 